\title{
Machine Learning-Based in-band OSNR Estimation from Optical Spectra
}

\author{
Fabiano Locatelli, Konstantinos Christodoulopoulos, Michela Svaluto Moreolo, Senior Member, \\ IEEE, Josep M. Fàbrega, Senior Member, IEEE, Salvatore Spadaro, Senior Member, IEEE
}

\begin{abstract}
Measuring the optical signal to noise ratio (OSNR) at certain network points is essential for failure handling, for single connection but also global network optimization. Estimating OSNR is inherently difficult in dense wavelength routed networks, where connections accumulate noise over different paths and tight filters do not allow the observation of the noise level at signal sides. We propose an in-band OSNR estimation process, which relies on a machine learning (ML) method, in particular on Gaussian process (GP) or support vector machine (SVM) regression. We acquired high-resolution optical spectra, through an experimental setup, using a Brillouin optical spectrum analyzer (BOSA), on which we applied our method and obtained excellent estimation accuracy. We also verified the accuracy of this approach for various resolution scenarios. To further validate it, we generated spectral data for different configurations and resolutions through simulations. This second validation confirmed the estimation quality of the proposed approach.
\end{abstract}

Index Terms-Machine learning, optical performance monitoring, optical signal to noise ratio, optical spectrum.

\section{INTRODUCTION}

$\mathrm{T}$ HE optical signal to noise ratio (OSNR) is considered one of the most important signal quality parameters to measure. It is transparent to the bit rate and modulation format and it can be easily correlated to the BER [1]. One of the most common method to measure the OSNR employs optical spectrum analyzers (OSAs) [2]. By interpolating the noise level at the sides of the considered channel, the OSA allows the measurement of the amplified spontaneous emission (ASE) noise introduced by the optical amplifiers and other noisesensed impairments. Such measurements are typically taken offline to optimize a newly deployed connections or for troubleshooting failures.

Issues arise in wavelength switched optical networks employing ultra-dense wavelength division multiplexing (ultraDWDM) or flex-grid filters [3]. In such networks, the channels exhibit different noise levels, according to their routes. Furthermore, a connection along its path crosses certain reconfigurable optical add/drop multiplexers (ROADMs), which employ optical filters. The filters introduce a sharp power drop between the channels, making the measurement of the noise level challenging [4]. Figure 1 shows two acquisition examples of filtered channels where it is apparently difficult to identify the noise level. Another issue is the filter cascade effect (FCE): after several filters the pass-band tightens, distorting the signal and making even harder the identification of the noise level [5]. Thus, measuring the OSNR has to be done in-band [3]. Since the introduction of coherent receivers polarization multiplexed (PM) channels are mostly used, making polarization nulling techniques for measuring OSNR unsuitable. A method is to establish the connection, measure the signal and turn it off to measure its noise. However, this cannot be done while the network is operating. Failure handling and dynamic network optimization in low margin and/or in disaggregated networks requires to measure the OSNR in-band and non-intrusively, as the network operates [6], [7].

Nowadays, very high-resolution optical spectrometry equipment are available, as for example the Brillouin optical spectrum analyzer (BOSA) [8]. This device exploits the stimulated Brillouin scattering (SBS), a non-linear optical effect that causes a very narrow filtering [9], which allows the BOSA to achieve spectral resolutions up to $0.1 \mathrm{pm}(12.5 \mathrm{MHz})$. On the other hand, the classic OSAs range in the order of 0.01 $\mathrm{nm}(1.25 \mathrm{GHz})$ [10]. Authors in [11] proposed to use a highresolution spectrum analyzer for in-band OSNR monitoring. However, such equipment is bulky and expensive, thus hard to be used in deployed networks, in the wild. Much cheaper and less accurate solutions, referred to as channel monitors [12], have also recently become available, and could potentially be used for in-band OSNR monitoring at ROADM nodes. However, it is unclear which spectral resolution and what processing method must be used to achieve good accuracy.

Machine learning (ML) has recently been adopted in several scientific fields and is also becoming attractive in optical

M. Svaluto Moreolo, and J. M. Fàbrega are with the Centre Tecnològic de Telecomunicacions de Catalunya, Optical Networks and Systems Department, $08860 \quad$ Castelldefels, Spain (email: michela.svaluto@cttc.es; josep.maria.fabrega@cttc.es).

S. Spadaro is with the Department of Signal Theory and Communications, Universitat Politecnica de Catalunya (UPC), 08034 Barcelona, Spain (e-mail: spadaro@tsc.upc.edu).
This work was partially funded by the ONFIRE project supported by EU Horizon 2020 research and innovation progr

F. Locatelli is with Nokia Bell Labs, 70435 Stuttgart, Germany, and also with the Centre Tecnologic de Telecomunicacions de Catalunya, Optical Networks and Systems Department, 08860 Castelldefels, Spain (e-mail: fabiano.locatelli@cttc.es).

K. Christodoulopoulos is with Nokia Bell Labs, 70435 Stuttgart, Germany (e-mail: konstantinos.1.christodoulopoulos@nokia-bell-labs.com). 


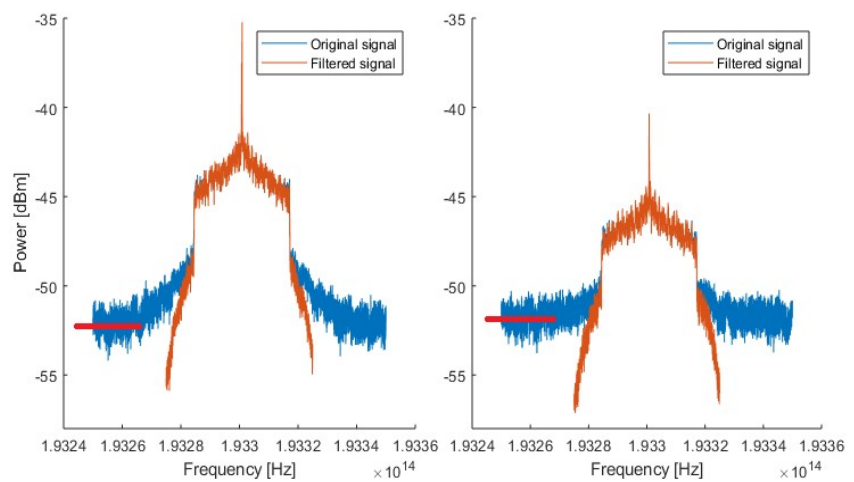

Fig. 1. Optical spectra before (blue) and after (orange) the $50 \mathrm{GHz}$-bandwidth filter captured at high-resolution $(0.1 \mathrm{pm})$ in the experimental setup. The red lines indicate the noise level floor.

communications. In [13], the authors considered four common ML models, and in particular, support vector machine (SVM), artificial neural network (ANN), k-nearest neighbors (KNN) and decision tree, and identified SVM as the most promising approach for OSNR estimation. However in [13], most of the spectral data were generated with a simulation tool and only few with experiments. Moreover, they considered classification with $1 \mathrm{~dB}$ accuracy, which is rather coarse, depending on the use case at hand. Finally, they processed wide and not in-band spectrum, which is not available in deployed filtered networks.

In this letter, we propose a method that, despite the aforementioned challenges, estimates accurately the OSNR from the in-band optical spectrum in short-distance scenarios. Indeed, for longer distance applications, the contribution given by the nonlinearities to the OSNR should be also considered [14]. We used a BOSA to capture high-resolution experimental spectral data, and in turn train two ML regression methods for estimating in-band OSNR: a Gaussian Model (GM) and an SVM model. Relying on high resolution optical spectra theoretically allows the identification of the channel noise level more precisely with respect to a standard OSA [6]. To evaluate the effect of optical spectra resolution on the estimation accuracy, we applied the same methods with lower-resolution optical spectral input and compared the results. Finally, we carried out a further validation of the proposed ML-based process using simulation-generated optical spectra with different modulation formats and various filter and noise scenarios. The proposed GM ML method achieved a maximum error of $1.1 \mathrm{~dB}$ in all the experimental scenarios, where OSNR ranged from 10 to $30 \mathrm{~dB}$, and a maximum error of $0.3 \mathrm{~dB}$ in all the simulated scenarios, where OSNR ranged from 22 to $35 \mathrm{~dB}$.

\section{EXPERIMENTAL SETUP AND SPECTRAL PROCESSING}

Figure 2 depicts the experimental setup used to capture several high-resolution optical spectra. We generated a $28 \mathrm{GBd}$ polarization multiplexed-quadrature phase shift keying (PMQPSK) modulated signal, with a tunable laser working at $1550.918 \mathrm{~nm}$. We obtained back to back (B2B) measurements and transmitted the signal over 4 different distance paths: 35 $\mathrm{km}, 50 \mathrm{~km}, 150 \mathrm{~km}$ and $200 \mathrm{~km}$, using the ADRENALINE testbed. At the output of the testbed, we placed a variable optical attenuator (VOA) and then an EDFA operating at

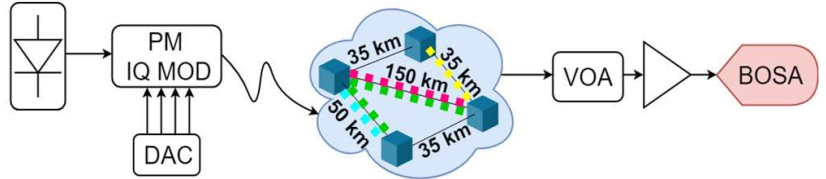

Fig. 2. Schematic diagram of the experimental setup for the high-resolution optical spectra acquisition. PM-IQ-MOD: polarization multiplexed-IQmodulator, DAC: digital-to-analog converter, VOA: variable optical attenuator, BOSA: Brillouin optical spectrum analyzer.

constant power to emulate more spans and obtained 16 different OSNR levels. Finally, we acquired the spectra using the BOSA. For each scenario, we collected a total of 160 optical spectra, specifically 10 for each VOA level (5 for each polarization state). Every time the optical signal enters or exits the ADRENALINE testbed, it also passes through an optical filter. In our case, the $35 \mathrm{~km}$ and $50 \mathrm{~km}$ scenarios include optical filters with $100 \mathrm{GHz}$-bandwidth, while in the $150 \mathrm{~km}$ and 200 $\mathrm{km}$ scenarios the signals entered and exited the testbed passing through a $100 \mathrm{GHz}$ and $50 \mathrm{GHz}$-bandwidth filter, respectively.

We then processed the collected spectra. During this phase, we applied a $50 \mathrm{GHz}$-bandwidth optical filter to the acquired spectra. This was done so as to create a variety of possible realistic network conditions, such as: laser drift and filter tightening, by misaligning the filter with the laser and reducing the size of the filter, respectively. Then after applying the filter, we cut the spectra at the filter edges to replicate a real DWDM spectrum, where each channel is bounded by its adjacent, thus resulting in a narrow area for measuring the OSNR. Figure 1 shows examples of high-resolution filtered optical spectra together with their original pre-filtered versions. As expected, the filter affected the noise outside the signal bandwidth, making sometimes infeasible to identify its actual level.

We represent the acquired optical spectrum with the vector $\boldsymbol{S}$ of length $n$. The length $n$ depends on the equipment spectral resolution $r(\mathrm{GHz})$ and on the network allocated bandwidth $b$ $(\mathrm{GHz})$, which corresponds to the configuration of the filters along the path, so that $n=b / r$. When measuring with the BOSA at high-resolution $(r=12.5 \mathrm{MHz})$ and for a filter bandwidth $b=50 \mathrm{GHz}$, the length $n$ was equal to 4000 . To examine the accuracy of the proposed OSNR estimation method, described in the next Section, in the case of an OSA or a channel monitor, i.e. with a resolution of $r=1.25 \mathrm{GHz}$, we post-processed the collected high-resolution spectra to create low-resolution versions. To do so, we averaged the spectra in the linear domain, reducing their length to $n=40$. Theoretically, we expect a higher accuracy with higherresolution optical spectra. We then associated each spectrum $\boldsymbol{s}$ to its reference OSNR value $y$, which was calculated through the integral method on the high-resolution spectra before the filter application. Spectra with OSNR reference values lower than $8 \mathrm{~dB}$ were excluded a priori from further processing phases, since in real systems such low OSNR signals would not be kept in operation. 


\section{PROPOSED ML OSNR ESTIMATOR}

Our goal is to find the mapping $f$ between the connection's spectrum $\boldsymbol{s}$ and its OSNR value $y$, that is $y=f(\boldsymbol{s})$. We denote with the matrix $\boldsymbol{S}_{\boldsymbol{c}}$, of dimensions $n \times m$, the set of $m$ collected spectra of signals with the same parameters $c=(r, b, q)$, where $r$ is the spectral resolution, $b$ is the filtered bandwidth and $q$ is the connection symbol rate. We also denote by vector $\boldsymbol{y}_{\boldsymbol{c}}$, of length $m$, their reference OSNR values. To approximate the estimation function $f$, we implemented a ML model $Q_{c}$ specific for channels with parameters $c$. Thus, we trained $Q_{c}$ with the sets $\left(\boldsymbol{S}_{\boldsymbol{c}}, \boldsymbol{y}_{\boldsymbol{c}}\right)$ as input. Let $\widehat{\boldsymbol{y}}_{\boldsymbol{c}}=Q_{c}\left(\boldsymbol{S}_{\boldsymbol{c}}\right)$ be the estimated OSNR values and $\boldsymbol{\varepsilon}_{\boldsymbol{c}}=\widehat{\boldsymbol{y}}_{\boldsymbol{c}}-\boldsymbol{y}_{\boldsymbol{c}}$ the estimation error. The goal of training is to identify $Q_{c}$ so as to minimize some function of the estimation error $\boldsymbol{\varepsilon}_{\boldsymbol{c}}$, for example the mean squared error (MSE).

SVM and GM are two nonparametric ML techniques for classification and regression, which rely on kernel functions. We formulated the estimation as a regression problem and we trained the SVM and GM models using the linear and the squared exponential kernel functions, respectively.

As mentioned, for each path and VOA configuration in the testbed, we collected a total of 10 spectra, 5 for each polarization state. To improve the quality of the considered spectral data, we first time-averaged the 5 spectra of each polarization state, and added up the 2 resulting spectra. Indeed, time averaging is a typical process to reduce the monitoring errors and the randomness of Gaussian effects. Furthermore, to reduce the effect of a laser drift, we identified the channel central frequency of each spectrum (detecting the peak relative to the carrier) and aligned them based on that.

The proposed ML-based estimation method requires for training the reference OSNR values. We described above how we obtained the reference OSNR values in the testbed. In operating networks, assuming deployed channel monitors at the nodes, we could measure the in-band OSNR of PM signals with the On/Off method [3] during their provisioning, before the channel operates. We can make use of the SNR monitored at the DSP of the coherent receiver after making certain assumptions and converting it to OSNR. We can also perform experiments in the lab to complement the above. Once the ML algorithm is trained with the spectra $\boldsymbol{S}_{c}$ and their reference OSNR values $\boldsymbol{y}_{c}$, it estimates the OSNR $\hat{y}$ of an operating channel with the same parameters $c$ from its spectrum $\boldsymbol{s}$.

\section{RESUlTS AND DisCUSSION}

We evaluated the estimation performance of the proposed ML method (Section III) using the high resolution spectra acquired in the experimental setup (Section II). To be more specific, all acquired spectra comprised the set $\boldsymbol{S}_{\boldsymbol{c}}$ with parameters $c=(r=12.5 \mathrm{MHz}, b=50 \mathrm{GHz}, q=28 \mathrm{GBd})$. The total number of spectra $m$ was 198 , and we used the $\sim 85 \%$ (169) of these to train the algorithm, whereas the remaining $\sim 15 \%$ (29) for testing it. To evaluate the estimation accuracy, we randomly shuffled the training and testing sets 200 times, trained a different ML model each time and tested it with the corresponding sets. In the first part of this section we report the results of the best performing ML model, which was GM.

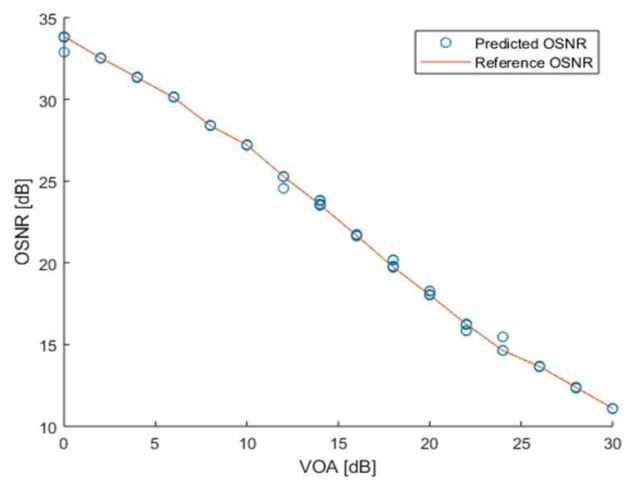

Fig. 3. Reference and predicted OSNR values as function of the VOA levels for the $50 \mathrm{~km}$ distance scenario.

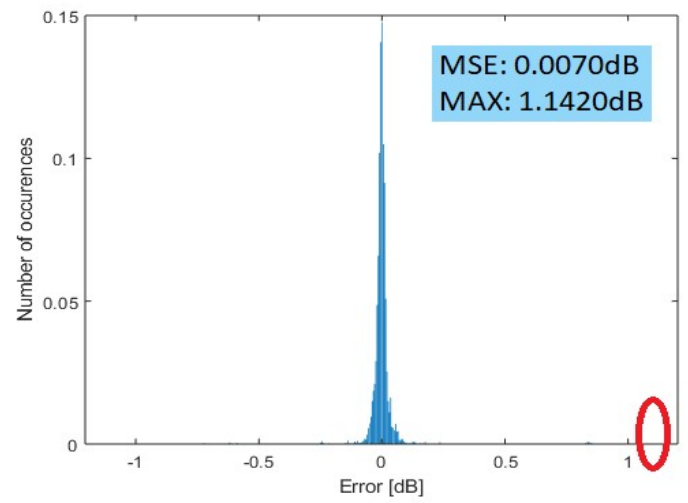

Fig. 4. Probability density function of the OSNR estimation error of the GM model and high-resolution spectra. The maximum error is highlighted in the red circle.

Figure 3 shows the reference and the estimated OSNR values for the $50 \mathrm{~km}$ path distance scenario as a function of the different VOA levels. We trained the GM ML model with the high resolution spectra training set with all the path distances (and B2B) and plot the spectra of the $50 \mathrm{~km}$ signals from the testing set. Figure 4 shows the probability density function (PDF) of the error made by the GM with respect to the reference for the high-resolution optical spectra. As highlighted in the figure inset, the mean squared error (MSE) was $0.0070 \mathrm{~dB}$ and the maximum error (MAX) was $1.1420 \mathrm{~dB}$. The accuracy achieved with the low-resolution spectra (which correspond to different parameters $c$ and a different trained model $Q_{c}$ ), were identical to those of the high-resolution case. Therefore, concerning the experimental acquired optical spectra, no difference arose between the two resolution versions. We did not observe any dependence of the estimation error with respect to the reference OSNR in the range between $10 \mathrm{~dB}$ to $30 \mathrm{~dB}$ of the experimentally acquired data. It is worth noting that the reference OSNR and the spectra used in the ML method were acquired with a state of the art measuring equipment (BOSA) with a dynamic range of $>80 \mathrm{~dB}$.

For a further validation, we carried out several VPI-based simulations and collected additional sets of optical spectra. Figure 5 shows the VPI simulation setup. We created a $28 \mathrm{GBd}$ PM-QPSK signal, yielding a $112 \mathrm{~Gb} / \mathrm{s}$ connection. A second order Gaussian optical filter was used to emulate the effect of passing through a number of ROADMs. We considered 16 VOA levels and two filter bandwidths: $37.5 \mathrm{GHz}$ and $50 \mathrm{GHz}$. 


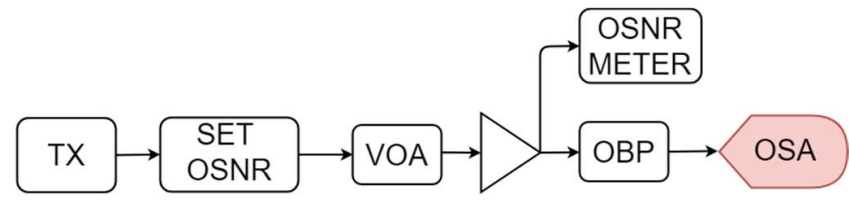

Fig. 5. Schematic diagram of the VPI simulation setup. TX: optical transmitted signal, VOA: variable optical attenuator, EDFA: erbium-doped fiber amplifier, OBP: optical bandpass filter, OSA: optical spectrum analyzer.

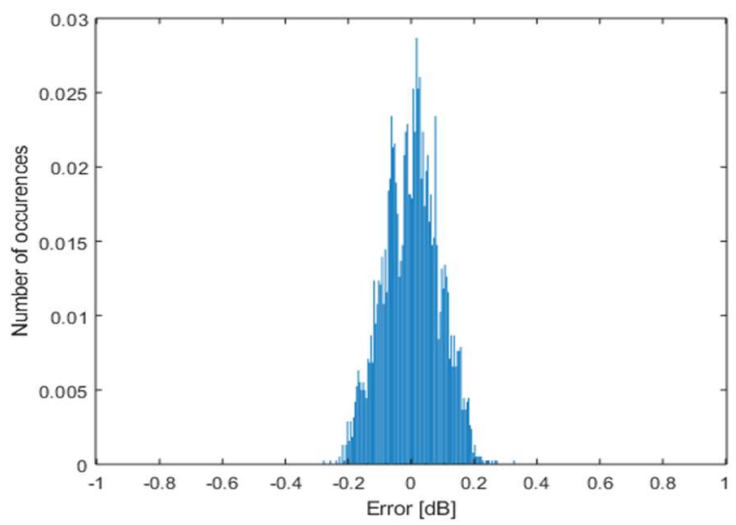

Fig. 6. Probability density function of the error committed by the GM while predicting the OSNR using PM-16QAM as modulation format for the highresolution VPI simulated spectra.

TABLE I

SUMMARY RESULTS FOR THE SIMULATED OPTICAL SPECTRA

\begin{tabular}{|c|c|c|c|c|c|c|}
\hline \multirow{2}{*}{$\begin{array}{c}\text { Modulation } \\
\text { format }\end{array}$} & \multirow{2}{*}{$\begin{array}{c}\text { Optical } \\
\text { filter } \\
\text { band- } \\
\text { width } \\
{[\text { GHz] }}\end{array}$} & \multirow{2}{*}{$\begin{array}{l}\text { Optical } \\
\text { spectral } \\
\text { resolution }\end{array}$} & \multicolumn{2}{|c|}{$\begin{array}{c}M S E[d B] \\
\left(\times 10^{-3}\right)\end{array}$} & \multicolumn{2}{|c|}{$\begin{array}{c}M A X[d B] \\
\left(\times 10^{-3}\right)\end{array}$} \\
\hline & & & $G M$ & $S V M$ & $G M$ & $S V M$ \\
\hline \multirow{4}{*}{$\begin{array}{l}\text { PM- } \\
\text { QPSK }\end{array}$} & \multirow{2}{*}{37.5} & $0.1 \mathrm{pm}$ & 8.5 & 21.6 & 212 & 495 \\
\hline & & & 6 & 8. & & 315 \\
\hline & \multirow{2}{*}{50} & $0.1 \mathrm{p}$ & 5.2 & 7.9 & 209 & 327 \\
\hline & & $0.01 \mathrm{~nm}$ & 5.4 & 9.1 & 215 & 302 \\
\hline \multirow{2}{*}{$\begin{array}{l}\text { PM- } \\
\text { 16QAM }\end{array}$} & \multirow{2}{*}{37.5} & $0.1 \mathrm{pm}$ & 7.7 & 20.2 & 327 & 547 \\
\hline & & $0.01 \mathrm{~nm}$ & 3.9 & 16.3 & 186 & 552 \\
\hline
\end{tabular}

In addition, we modified the filter central frequency and bandwidth to emulate two realistic scenarios: filters shift/laser drift $( \pm 1 \mathrm{GHz}$ ), and FCE (reducing the $37.5 \mathrm{GHz}$ filter to 25 $\mathrm{GHz}$ ). We collected the spectral data by an OSA-VPI module with $0.1 \mathrm{pm}$ and $0.01 \mathrm{~nm}$ resolutions, and in total we acquired 144 optical spectra for each resolution. Exploiting the same VPI setup, we also generated a $224 \mathrm{~Gb} / \mathrm{s}$ PM-16QAM signal, but we only considered the $37.5 \mathrm{GHz}$ filter configuration. We collected 128 spectra for PM-16QAM for each resolution scenario. In total we created six sets of spectra according to the related parameters $c$ : high and low-resolution for two filter bandwidths (37.5 and $50 \mathrm{GHz}$ ) for PM-QPSK, and high and low-resolution with $37.5 \mathrm{GHz}$ filter for PM-16QAM. As before, for each set we used $85 \%$ of the spectra for training and the $15 \%$ for testing. We shuffled the spectra of each set 200 times, each time we trained, estimated, and obtained the errors. Figure 6 shows the estimation error PDF with high-resolution spectra and the PM16QAM channel. We summarize all the results for both the ML models in Table I. We observed a maximum OSNR estimation error lower than $0.4 \mathrm{~dB}$ in all the considered scenarios. Again, as in the experimental results, minor deviations were observed when comparing the two resolution versions of the spectra.

\section{CONCLUSION}

We developed a machine learning-based in-band OSNR estimator, relying on GM or SVM models. We evaluated its estimation accuracy with experimental and simulation generated spectra. The results showed an excellent accuracy of the proposed process, a maximum error of $1.1 \mathrm{~dB}$ in experimental and $0.3 \mathrm{~dB}$ in simulated scenarios.

\section{ACKNOWLEDGEMENT}

The authors would like to thank Aragon Photonics Labs for providing the BOSA used for the experiments.

\section{REFERENCES}

[1] C. C. K. Chan, Optical Performance Monitoring. Burlington, MA: Elsevier, 2010.

[2] Optical signal-to-noise ratio measurement for dense wavelengthdivision multiplexed systems, IEC 61280-2-9, 2009.

[3] Fibre Optic Communication System Design Guides - Part 12: InBand Optical Signal-To-Noise Ratio (OSNR), IEC TR 61282-12, 2016.

[4] A. Morea, J. Renaudier, T. Zami, A. Ghazisaeidi, and O. BertranPardo, "Throughput Comparison Between 50-GHz and 37.5-GHz Grid Transparent Networks [invited]," IEEE/OSA J. Opt. Commun. Netw., vol. 7, no. 2, pp. A293-A300, Feb. 2015.

[5] J. M. Fàbrega et al., "On the filter narrowing issues in elastic optical networks," IEEE/OSA J. Opt. Commun. Netw., vol. 8, no. 7, pp. A23-A33, Jul. 2016.

[6] Viavi Whitepaper, "In-Band OSNR Measurements on 40G Polarization Multiplexed QPSK Signals Using a FieldDeployable, High-Resolution OSA," Viavi Solutions Inc., 2015. [Online]. Available: www.viavisolutions.com/enus/literature/band-osnr-measurements-40-g-polarizationmultiplexed-qpsk-signals-using-field-deploy-white-paper-en.pdf

[7] Z. Huang et al., "A Novel In-Band OSNR Measurement Method Based on Normalized Autocorrelation Function," IEEE Photon. $J$., vol. 10, no. 2, Apr. 2018.

[8] A. Photonics, "BOSA Technology," [Online]. Available: https://aragonphotonics.com/bosa/, accessed July 2019.

[9] J. M. Subias Domingo et al., "Very High Resolution Optical Spectrometry by Stimulated Brillouin Scattering," IEEE Photon. Technol. Lett., vol. 17, no. 4, pp. 855-857, Apr. 2005.

[10] J. M. Fàbrega, M. Svaluto Moreolo, and L. Nadal, "Optical Performance Monitoring Systems in Disaggregated Optical Networks," in Proc. Int. Conf. Transp. Opt. Netw. (ICTON), Bucharest, Romania, Jul. 2018, pp. 1-4.

[11] W. Moench and E. Loecklin, "Measurement of Optical Signal-toNoise-Ratio in Coherent Systems using Polarization Multiplexed Transmission," in Proc. Opt. Fiber Commun. Conf. Exhib., Los Angeles, CA, USA, 2017, pp. 1-3.

[12] "Flexgrid High Resolution Optical Channel Monitor (OCM)," Finisar, 2015. [Online]. Available: www.finisar.com/sites/default /files/downloads/flexgrid_high_resolution_ocm_pb_web.pdf

[13] D. Wang et al., "Machine Learning-Based Multifunctional Optical Spectrum Analysis Technique," IEEE Access., vol.7, pp. 19726-19737, Jan. 2019.

[14] M. Lonardi et al., "Optical Nonlinearity Monitoring and Launched Power Optimization by Artificial Neural Networks," to appear, European Conf. Optical Communication, Sep. 2019. 